\title{
Intraoperative measurement of thyroglobulin in lymph node aspirates for the detection of metastatic papillary thyroid carcinoma
}

This article was published in the following Dove Press journal:

OncoTargets and Therapy

II September 2017

Number of times this article has been viewed

\section{Yizeng Wang \\ Yuanchao Liu \\ Xiaoning Wang \\ Xin Li \\ Ruoyu Jiang \\ Xianghui He}

Department of General Surgery, Tianjin Medical University General Hospital, Tianjin Medical University, Tianjin, People's Republic of China

Correspondence: Xianghui He Department of General Surgery, Tianjin Medical University General Hospital, Tianjin Medical University, 154 Anshan Road, Tianjin 300052, People's Republic of China Tel +862260363970 Email humphreyhe@163.com
Background: Among patients with papillary thyroid carcinoma (PTC), 30\%-80\% have cervical lymph node (LN) metastases, which are most commonly located in the central compartment. However, preoperative ultrasonography identifies malignant central compartment LNs in only $20 \%-30 \%$ of cases. We aimed to evaluate the diagnostic value of intraoperative thyroglobulin (Tg) measurement in fine-needle aspirates (FNA-Tg) of suspicious metastatic LNs.

Methods: In total, 75 patients $(75 \mathrm{LNs})$ with PTC or suspected PTC were enrolled in this study. Suspicious metastatic LNs were isolated intraoperatively, and FNA-Tg was performed. Then, the $\mathrm{Tg}$ values were compared with the corresponding pathological results and preoperative ultrasonography.

Results: In total, 37 LNs were diagnosed as malignant, and 38 were benign. According to the receiver operating characteristic (ROC) curve, the optimal cutoff value of intraoperative FNA-Tg was $147.5 \mathrm{ng} / \mathrm{mL}$ (sensitivity, $81.1 \%$; specificity, $100 \%$; $p=0.000$ ). The sensitivity and specificity for detecting central compartment LN metastasis were $77.78 \%$ (21/27) and 100\% (36/36), respectively. The corresponding sensitivity of preoperative ultrasonography was lower than that of FNA-Tg $(p=0.000)$. Serum Tg-antibody $(\mathrm{Ab})$, thyroid-stimulating hormone (TSH) and thyroid peroxidase antibody (TPO-Ab) were not significantly associated with FNA-Tg values. There was no statistical correlation between preoperative serum $\mathrm{Tg}$ and intraoperative FNA-Tg $(p=0.451)$.

Conclusion: Intraoperative FNA-Tg levels of suspicious metastatic cervical LNs can be useful for diagnosing metastatic PTC. Intraoperative LN-FNA-Tg may have an important role in determining which surgical procedure to perform.

Keywords: papillary thyroid carcinoma, lymph node metastasis, intraoperative thyroglobulin measurement, fine-needle aspirates, preoperative ultrasonography

\section{Introduction}

Papillary thyroid carcinoma (PTC), a subset of differentiated thyroid carcinoma (DTC), is the most common malignant endocrine tumor, and the incidence of PTC has continually increased worldwide over the past few decades. PTC has a remarkable tendency to metastasize to the cervical lymph nodes (LNs). Thyroidectomy with or without dissection of the cervical LNs is the first choice of treatment for PTC. ${ }^{131} \mathrm{I}$ and thyroid hormone suppression therapy are followed according to the disease stages. Therapeutic LN dissection for thyroid cancer nodal metastases is recommended. However, prophylactic central compartment neck dissection for PTC remains controversial. ${ }^{1}$

Cervical LN metastasis (LNM) occurs in $30 \%-80 \%$ of PTC cases, ${ }^{2,3}$ with the primary metastasis most commonly located in the central compartment (ie, central 
compartment LN or CCLN). ${ }^{4}$ Although it appears to have no significant impact on overall survival, cervical LNM is an independent risk factor for local recurrence. ${ }^{5}$ Preoperative ultrasonography of the neck is the main approach for staging malignant thyroid nodules. ${ }^{1}$ The sensitivity of ultrasonography in the diagnosis of LNM of the central compartment is lower than that of LNM of the lateral neck due to the impact of the thyroid gland. Preoperative ultrasonography, including high-resolution ultrasonography, identifies malignant CCLNs in 20\%-30\% of cases. ${ }^{6,7}$ Computed tomography (CT), magnetic resonance imaging (MRI) and positron emission tomography (PET)-CT can be used as complementary methods for detecting cervical LNM. The sensitivities of MRI and PET are relatively low. ${ }^{8}$ Some studies have revealed that the sensitivity of CT in combination with ultrasonography is higher than that of ultrasonography alone, ${ }^{9,10}$ but the presence of thyroid tissue limits the diagnostic performance of imaging the central compartment.

Despite the preoperative imaging, inspection of suspicious LNs in the central compartment during thyroidectomy is still needed. ${ }^{11}$ Since 1992, thyroglobulin (Tg) measurement in fine-needle aspirates (FNA-Tg) has been proposed to detect malignant cervical LNs preoperatively, particularly for uncertain cases. ${ }^{12}$ Many studies have demonstrated the diagnostic benefit of measuring LN FNA-Tg to detect LNM of PTC.

In our study, suspicious metastatic cervical LNs were isolated during thyroidectomy. Then, FNA-Tg levels were measured, and pathological examination of these LNs was performed; the results of these tests and examinations were compared, including the results of the preoperative ultrasonography assessing the suspicious cervical LNs. The purpose of our study was to evaluate the diagnostic value of intraoperative FNA-Tg measurement of suspicious LNs for detecting LNM.

\section{Materials and methods}

\section{Patients}

This study included 77 patients (77 LNs) with PTC or suspected PTC diagnosed by intraoperative frozen section or cytology of preoperative FNA from November 2014 to December 2016 in Tianjin Medical University General Hospital (Tianjin, People's Republic of China). In total, two LNs were excluded due to confirmation of presence of thyroid tissue (one LN) and parathyroid tissue (one LN) by pathology. Overall, 75 patients with 75 LNs were eligible. Serum Tg, anti-Tg antibody (Tg-Ab), thyroid-stimulating hormone (TSH) and thyroid peroxidase antibody (TPO-Ab) were measured preoperatively. This study was approved by the institutional review board of Tianjin Medical University General Hospital. Written informed consent was obtained from all patients prior to operation and for this study.

\section{Procedure and measurement of FNA-Tg}

Every patient was in the supine position with his or her neck extended. The thyroid surgery was performed under general anesthesia with endotracheal intubation. When thyroid lobectomy was complete, lesions were immediately sent for intraoperative frozen section pathology. Ipsilateral CCLN dissection was followed when the diagnosis was PTC or suspected PTC, and lateral compartment LN dissection was performed only in patients with suspected lateral compartment LNM. A most suspicious metastatic LN was isolated, and FNA-Tg was performed immediately after excision of the LN. The features that were suggestive of a metastatic LN were as follows: enlargement, a round shape rather than an oval shape (long/transverse diameter ratio of $<1.5$ ), cystic change and hard texture. FNA was performed with a freehand technique using a 23-gauge needle with a $5 \mathrm{~mL}$ syringe; two passes from different regions were performed with each aspiration. Each pass lasted no more than 5 seconds, with the needle moving back and forth within the nodule $\sim 3$ times per second. The needle and syringe were washed immediately with $1 \mathrm{~mL}$ of normal saline solution, and the washout fluid was sent to the laboratory for $\mathrm{Tg}$ assays. Then, the aspirated LN was sent for pathological examination. The $\mathrm{Tg}$ values were compared with the pathology results ultimately.

\section{Measurement of serum $\mathrm{Tg}, \mathrm{Tg}-\mathrm{Ab}, \mathrm{TSH}$, TPO-Ab and FNA-Tg}

Serum $\mathrm{Tg}, \mathrm{Tg}-\mathrm{Ab}$ and TSH were measured within a period of 2 weeks before surgery. A TSH assay (reference 0.30-5.00 $\mu \mathrm{IU} / \mathrm{mL}$, maximum $150.00 \mu \mathrm{IU} / \mathrm{mL}$ ) was performed on a fully automated ADVIA Centaur analyzer (Siemens Healthineers, Erlangen, Germany). Serum $\mathrm{Tg}$ and FNA-Tg (reference $0-55.00 \mathrm{ng} / \mathrm{mL}$, maximum $300.00 \mathrm{ng} / \mathrm{mL}$ ), Tg-Ab (reference $0-40.00 \mathrm{IU} / \mathrm{mL}$, detection range $20-3,000.00 \mathrm{IU} / \mathrm{mL}$ ) and $\mathrm{TPO}-\mathrm{Ab}$ (reference 0-35.00 IU/mL, detection range 10-3,000.00 IU/mL) were measured on a fully automated IMMULITE 2000 analyzer (Siemens Healthineers). These assays were based on the chemiluminescent reaction principle.

\section{Pathological diagnosis}

The diagnosis of cervical LN metastasis from paraffin sections is the gold standard. Therefore, the diagnosis of cervical 
LN metastasis of PTC was defined as a malignant LN. When there was no evidence of metastasis in the paraffin sections, the LN was defined as benign.

\section{Preoperative ultrasonography}

Preoperative ultrasonography was performed using MyLab ${ }^{\mathrm{TM}}$ ClassC (Esaote, Genova, Italy) several days before surgery by a specialized group of radiologists at the Department of General Surgery, Tianjin Medical University General Hospital. The appearance of suspicious metastatic LNs included the following features: enlargement, a rounded shape, irregular pattern, the absence of fatty hilum, cystic change, calcifications and peripheral vasculature.

\section{Data analysis}

According to the pathological results of the examined LNs, patients were divided into two groups: the malignant LN group, in which the examined node had metastasis, and the benign LN group, in which the examined node did not have metastasis. In the present study, the levels of FNA-Tg, serum Tg, Tg-Ab, TSH and TPO-Ab that were more than the maximum or less than the minimum were marked as the maximum or minimum, respectively.

Continuous variables were expressed as the medians (interquartile range). The Mann-Whitney $U$-test was used to compare intraoperative FNA-Tg, preoperative serum $\mathrm{Tg}$, $\mathrm{Tg}-\mathrm{Ab}$, TPO-Ab and TSH between the two groups. Categorical variables were presented as ratios, and the chi-square test was used for comparisons. Spearman's rank correlation was used to analyze the correlation between preoperative serum $\mathrm{Tg}$ and intraoperative FNA-Tg. Receiver operating characteristic (ROC) curve analysis was performed to determine the optimal cutoff value of FNA-Tg for the diagnosis of LNM. The area under the ROC curve (AUROC) was also obtained with a confidence interval (CI). Diagnostic performances, such as sensitivity, specificity of intraoperative LN-FNA-Tg and preoperative ultrasonography, were evaluated by comparing the results with the corresponding LN pathology of the paraffin section and were compared using the chi-square test or the Fisher's exact test. All $p$-values were two sided, and data with $p$-values $<0.05$ were considered statistically significant. All analyses were performed with SPSS software 17.0 (IBM, Armonk, New York, USA).

\section{Results}

\section{Patient characteristics}

All 75 patients (75 LNs) eligible for this study were diagnosed with PTC by final histology. The mean age of these patients was 46.87 years $(46.87 \pm 12.76$ years), and
$61(81.33 \%)$ were females. Only one patient had previous PTC surgery. According to the 7th American Joint Committee on Cancer (AJCC) tumor, node and metastases (TNM) system, there were 11 pT1 patients, two pT2 patients, 61 pT3 patients (mainly due to minimal extrathyroidal extension) and one pT4 patient. There were 37 stage I patients, 30 stage III patients and eight stage IV patients. In total, 39 patients in the study had papillary thyroid microcarcinoma (PTMC). Among all 75 analyzed LNs, 37 LNs (49.33\%) were diagnosed as metastatic PTC, while 38 LNs (50.67\%) were diagnosed as benign. Totally, 63 CCLNs (84\%) were examined, including 27 malignant LNs and 36 benign LNs. Among the 12 lateral neck LNs, 10 and two LNs were diagnosed as malignant and benign, respectively.

The characteristics of both the malignant and benign LN groups are presented in Table 1. There were no statistically significant differences in age $(p=0.913)$, preoperative serum TPO-Ab $(p=0.157)$, serum TSH $(p=0.907)$ or serum $\operatorname{Tg}-\mathrm{Ab}(p=0.322)$ between the two groups. Gender $(p=0.021)$, primary tumor size $(p=0.000), \mathrm{LN}$ location $(p=0.01)$, serum Tg level $(p=0.005)$ and preoperative FNA-Tg level $(p=0.000)$ were significantly different between the two groups. The median serum $\mathrm{Tg}$ level was higher in the malignant LN group (30 ng/mL; interquartile range: $9.475-70.6 \mathrm{ng} / \mathrm{mL}$ ) than in the benign $\mathrm{LN}$ group $(10.7 \mathrm{ng} / \mathrm{mL}$; interquartile range: 3.843-25.2 ng/mL). The intraoperative FNA-Tg level was also higher in the malignant LN group than in the benign LN group, but no statistical correlation between the preoperative serum Tg and the intraoperative FNA-Tg was present according to Spearman's rank correlation (all patients: $p=0.451$; malignant LN group: $p=0.248$; benign LN group: $p=0.095$ ).

\section{Intraoperative LN-FNA-Tg level and final diagnosis of $\mathrm{LN}$}

There was a statistically significant difference in the intraoperative LN-FNA-Tg level between the two groups. The intraoperative FNA-Tg level of the malignant LN group (median: $300 \mathrm{ng} / \mathrm{mL}$; interquartile range: $139-300 \mathrm{ng} / \mathrm{mL}$ ) was much higher than that of the benign $\mathrm{LN}$ group (median: $19.62 \mathrm{ng} / \mathrm{mL}$; interquartile range: $6.163-39.875 \mathrm{ng} / \mathrm{mL}$ ). According to the ROC curve, the optimal cutoff value for intraoperative determination of LN-FNA-Tg was $147.5 \mathrm{ng} / \mathrm{mL}$; the AUROC was 0.927 , with a $95 \%$ CI of $0.863-0.99(p=0.000)$, sensitivity of $81.1 \%$ and specificity of $100 \%$. When the intraoperative LN-FNA-Tg value was $147.5 \mathrm{ng} / \mathrm{mL}$, the numbers of true-positive (TP), true-negative (TN), false-positive (FP) and false-negative (FN) results were 30, 38, zero and seven, respectively (Figure 1). The positive predictive value (PPV) was $100 \%$, and the negative predictive value (NPV) was $84.44 \%$. 
Table I Patient characteristics according to pathological results of LNs that underwent fine-needle aspirates

\begin{tabular}{|c|c|c|c|}
\hline Characteristics & Malignant LN group & Benign LN group & $p$-value \\
\hline Number of patients & 37 & 38 & - \\
\hline Number of LNs & 37 & 38 & - \\
\hline Gender, male/female, $\mathrm{n}$ & $12 / 25$ & $4 / 34$ & $0.021^{\mathrm{a}}$ \\
\hline Mean age, years & $46.7 \pm 12.732$ & $47.03 \pm 12.965$ & $0.913^{b}$ \\
\hline Tumor size, mm & $16.405 \pm 8.418$ & $9.776 \pm 5.373$ & $0.000^{\mathrm{b}}$ \\
\hline Multiplicity, yes/no, n & $15 / 22$ & $14 / 24$ & $0.742^{\mathrm{a}}$ \\
\hline Extracapsular invasion, yes/no, $\mathrm{n}$ & $33 / 4$ & $29 / 9$ & $\left.0.14\right|^{\mathrm{a}}$ \\
\hline Central/lateral cervical LNs, $\mathrm{n}$ & $27 / 10$ & $36 / 2$ & $0.0 \mathrm{I}^{\mathrm{a}}$ \\
\hline $\mathrm{pTI} / \mathrm{T} 2 / \mathrm{T} 3 / \mathrm{T} 4, \mathrm{n}$ & $2 / 2 / 32 / 1$ & $9 / 0 / 29 / 0$ & - \\
\hline AJCC stage I/II/III/IV, n & $15 / 0 / 14 / 8$ & $22 / 0 / 16 / 0$ & - \\
\hline PTMC, n & 13 & 26 & - \\
\hline Preoperative serum $\mathrm{Tg}, \mathrm{ng} / \mathrm{mL}$ & $30(9.475-70.6)$ & $10.7(3.843-25.2)$ & $0.005^{c}$ \\
\hline Preoperative serum $\mathrm{Tg}-\mathrm{Ab}, \mathrm{IU} / \mathrm{mL}$ & $20(20-20)$ & $20(20-25.13)$ & $0.322^{c}$ \\
\hline Preoperative serum TSH, $\mu \mathrm{IU} / \mathrm{mL}$ & $2.275(1.307-3.109)$ & $2.286(I .542-3.25 I)$ & $0.907^{c}$ \\
\hline Preoperative serum TPO-Ab, IU/mL & $10(10-51.65)$ & $12.2(10-163.25)$ & $0.157^{c}$ \\
\hline Intraoperative LN-FNA-Tg, ng/mL & $300(139-300)$ & $19.62(6.163-39.875)$ & $0.000^{c}$ \\
\hline
\end{tabular}

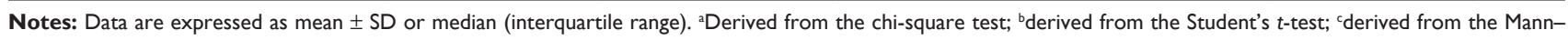
Whitney U-test. '-' indicates data not compared.

Abbreviations: AJCC, American Joint Committee on Cancer; FNA-Tg, thyroglobulin measurement in fine-needle aspirates; LN, lymph node; PTMC, papillary thyroid microcarcinoma; $\mathrm{Tg}$, thyroglobulin; Tg-Ab, anti-thyroglobulin antibody; TPO-Ab, thyroid peroxidase antibody; TSH, thyroid-stimulating hormone.

As for CCLNs, the numbers of TP, TN, FP and FN were 21, 36, zero and six, respectively (Figure 1); the corresponding results of sensitivity, specificity, PPV and NPV were $77.78 \%$, $100 \%, 100 \%$ and $85.71 \%$, respectively (Table 2 ).

In total, 39 patients with PTMC (13 patients in the malignant LN group and 26 patients in the benign LN group) were analyzed to determine the optimal cutoff value of intraoperative FNA-Tg for the assessment of malignant LN. The difference in intraoperative FNA-Tg levels between these two groups was statistically significant $(p=0.000)$. The median

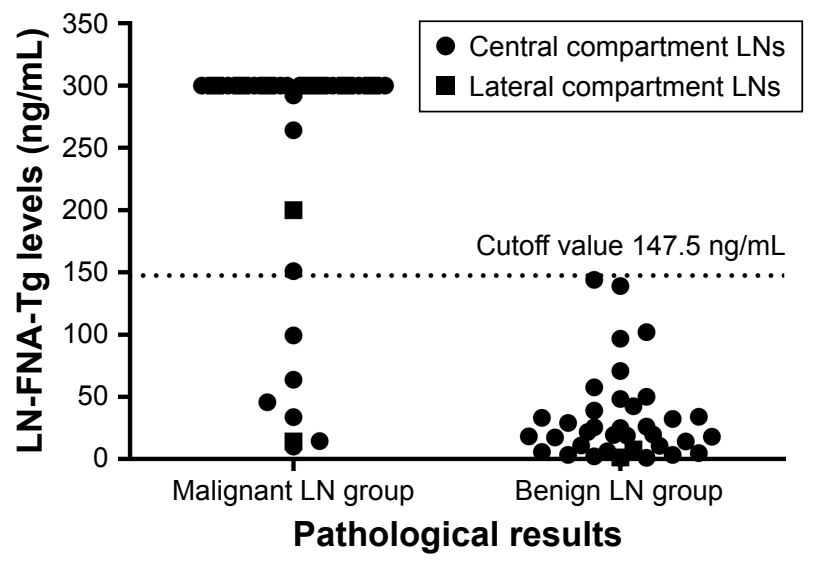

Figure I Distribution of intraoperative LN-FNA-Tg levels according to pathological results.

Notes: When the intraoperative LN-FNA-Tg value to determine malignant cervical LNs was $147.5 \mathrm{ng} / \mathrm{mL}$, the numbers of true-positive, true-negative, false-positive and false-negative results were 30,38 , zero and seven, respectively, and those of CCLNs were 21,36 , zero and six, respectively. The solid bar indicates malignant LN group was diagnosed pathologically as LN metastasis of PTC, while the benign LN group has no pathological evidence of $L N$ metastasis.

Abbreviations: CCLN, central compartment lymph node; LN, lymph node; FNA$\mathrm{Tg}$, thyroglobulin measurement in fine-needle aspirates. intraoperative FNA-Tg level of the malignant LN group (300 $\mathrm{ng} / \mathrm{mL}$ ) was higher than that of the benign LN group $(19.27 \mathrm{ng} / \mathrm{mL})$. According to the ROC curve, the optimal cutoff value for intraoperative LN-FNA-Tg was $98.1 \mathrm{ng} / \mathrm{mL}$; the AUROC was 0.929 , with a $95 \%$ CI of $0.832-1.000(p=0.000)$.

The sensitivity was $84.6 \%$, and the specificity was $96.2 \%$.

\section{Preoperative ultrasonography results}

According to the preoperative ultrasonography, 15 of the 75 patients had suspicious malignant cervical LNs, and four patients $(4 / 37,10.81 \%)$ had suspicious CCLNs (one patient with suspicious concurrent lateral cervical LNs). According to the histological results, 13 patients were diagnosed with PTC and cervical LNM, while two patients with suspicious malignant LNs of the lateral neck were diagnosed with PTC without cervical LNM. The sensitivity, specificity, PPV and NPV of preoperative ultrasonography in predicting malignant cervical LN were $35.14 \%$ (13/37), 94.74\% (36/38), 86.67\% (13/15) and 60\% (36/60), respectively. The sensitivity, specificity, PPV and NPV of preoperative ultrasonography for the assessment of malignant CCLN were $14.81 \%$ (4/27), $100 \%$ (36/36), 100\% (4/4) and $61.02 \%$ (36/59), respectively (Table 2 ).

\section{Discussion}

In 1992, Pacini et al ${ }^{12}$ were the first to demonstrate that $\mathrm{Tg}$ was detectable in the washout fluid of FNA cytology. High concentrations of FNA-Tg in the neck nodes outside the thyroid gland indicated the diagnosis of metastatic thyroid cancer, regardless of a previous history of thyroidectomy. ${ }^{12}$ 
Table 2 Comparison of diagnostic performances of intraoperative LN-FNA-Tg and preoperative ultrasonography to assess malignant cervical LN and CCLN

\begin{tabular}{lllllll}
\hline Subtypes & Procedures & Sensitivity & Specificity & PPV & NPV & Accuracy \\
\hline Cervical LN & LN-FNA-Tg & $81.1 \%$ & $100 \%$ & $100 \%$ & $84.44 \%$ & $90.67 \%$ \\
& Ultrasonography & $35.14 \%$ & $94.74 \%$ & $86.67 \%$ & $60 \%$ & $65.33 \%$ \\
& P-value & 0.000 & 0.493 & 0.755 & 0.262 & 0.000 \\
CCLN & LN-FNA-Tg & $77.78 \%$ & $100 \%$ & $100 \%$ & $85.71 \%$ & $90.48 \%$ \\
& Ultrasonography & $14.81 \%$ & $100 \%$ & $100 \%$ & $61.02 \%$ & $63.49 \%$ \\
& p-value & 0.000 & - & - & 0.273 & 0.000 \\
\hline
\end{tabular}

Note: '-' indicates data not compared.

Abbreviations: CCLN, central compartment lymph node; FNA-Tg, thyroglobulin measurement in fine-needle aspirates; LN, lymph node; PPV, positive predictive value; NPV, negative predictive value.

Since then, measurable $\mathrm{Tg}$ in the FNA washout fluids has proven to be more sensitive than fine-needle aspiration cytology (FNAC) in determining DTC recurrence in LNs and neck masses. ${ }^{13-15}$ Reported sensitivities for FNA-Tg vary from $81.4 \%$ to $100 \%$ according to the cutoff values, while they range from $55 \%$ to $85 \%$ for FNAC. ${ }^{13,16-18}$ FNA-Tg measurement is an accurate and useful tool to detect and differentiate cervical LNM from DTC. This study examined the value of intraoperative FNA-Tg of cervical LNs for the detection of metastatic PTC. To the best of our knowledge, this is the first study to perform LN-FNA-Tg intraoperatively for the diagnosis of lymphatic metastasis of PTC.

In the present study, the diagnostic capabilities of intraoperative LN-FNA-Tg and preoperative ultrasonography to assess malignant cervical LN were compared. The sensitivity and diagnostic accuracy were significantly different for these two methods $(p=0.000)$. The sensitivity of intraoperative LN-FNA-Tg was higher than that of preoperative ultrasonography ( $85.1 \%$ vs $35.14 \%$ ). There was no significant difference in terms of specificity, PPV or NPV between these two methods (Table 2). The results indicated that intraoperative LN-FNA-Tg could be a useful method to detect malignant LNs missed by preoperative ultrasonography.

Neck ultrasonography is the first-line imaging modality to detect suspicious malignant cervical LNs derived from PTC, but only $20 \%-30 \%$ of malignant CCLNs, which are the most common sites of metastasis, are identified preoperatively by ultrasonography. The sensitivity of preoperative ultrasonography for the assessment of malignant CCLN was $14.81 \%(4 / 27)$ in this study. A high risk of FP results exists when FNA and/or FNA-Tg was used preoperatively to diagnose the LNM of PTC, particularly for LNs located behind the thyroid tissue. To lower the FP rate, doctors should avoid puncturing thyroid tissue while performing FNA. ${ }^{19}$ In our study, FNA of suspicious malignant CCLNs was performed after thyroid lobectomy; thus the FP rate derived from intersecting thyroid tissue was lower. Preoperative ultrasonography identifies only half of the LNs found during surgery due to the presence of the overlying thyroid gland. Small-sized suspicious malignant CCLNs located behind the thyroid gland that are missed by preoperative ultrasonography may be found intraoperatively. Further, the FNA-Tg level of suspicious malignant CCLNs provides some information about the metastasis of PTC. In this study, when the cutoff value of LN-FNA-Tg was $147.5 \mathrm{ng} / \mathrm{mL}$, the sensitivity of intraoperative FNA-Tg for detecting malignant CCLNs was significantly higher than that of preoperative ultrasonography ( $77.78 \%$ vs $14.81 \%, p=0.000$ ), as was the diagnostic accuracy $(90.48 \%$ vs $63.49 \%, p=0.000)$.

During the thyroidectomy, thyroid lesions and/or suspicious LNs were primarily sent for intraoperative frozen section pathology. The sensitivity and specificity of frozen sections for thyroid lesions or extrathyroidal extension were $58 \%-94 \%$ and $93 \%-100 \%$, respectively. ${ }^{5,20-22}$ Advantages of using intraoperative frozen sections were the speed of processing and the high rate of correct diagnoses, but FN diagnoses continued to exist. FN diagnoses may result in reoperation, which has a higher risk of permanent hypoparathyroidism and recurrent laryngeal nerve injury. According to our study, intraoperative FNA-Tg was helpful for diagnosing LNM of PTC. FN diagnoses could be decreased if the suspicious LN is subjected to FNA-Tg determination intraoperatively for suspected metastasis and if the result of the FNA-Tg test is obtained immediately. Then, therapeutic rather than prophylactic - or no - neck dissection will be performed, avoiding the higher risk of reoperative complications and the cost of reoperation due to recurrence. Furthermore, intraoperative frozen sections require resected LNs, while FNA-Tg can be performed in vivo. The intraoperative FNA-Tg technique has auxiliary diagnostic value for LNM of PTC and may have greater value for some institutions where intraoperative frozen section pathology is not available.

Many molecular markers based on thyroid tissue (from operation or FNA) or peripheral blood, such as $B R A F, R E T /$ $P T C, R A S$ and $V E G F$ single-nucleotide polymorphisms (SNPs), have been studied or used in clinical practice in 
recent years to predict thyroid malignancy or prognosis. ${ }^{23,24}$ However, the available molecular markers have been used in a limited number of centers and require further verification. Despite the advances in molecular research, the malignancy of thyroid nodules that are reported as follicular neoplasms or suspected follicular neoplasms by FNAC cannot be reliably predicted preoperatively. A recent study of a large related clinical series showed that $26.2 \%$ of patients $(85 / 324)$ had complete thyroidectomy after hemithyroidectomy, and the definitive histological diagnosis was thyroid carcinoma (DTC was the most common type). ${ }^{25}$ We suppose that the intraoperative FNA-Tg of suspicious metastatic LNs may have supplementary value to diagnose LNM of DTC, to determine the optimal extent of resection and, thus, to reduce the possibility of reoperation for complete thyroidectomy.

The optimal cutoff value of intraoperative LN-FNA-Tg in our study was $147.5 \mathrm{ng} / \mathrm{mL}$, which was several times greater than the cutoff value of $32.04-36.00 \mathrm{ng} / \mathrm{mL}$ recommended by other studies in patients with or without an intact thyroid gland. ${ }^{26}$ This difference exists mainly because this study had more cytological materials than the others.

In addition, whether serum $\mathrm{Tg}$ - $\mathrm{Ab}$ affects the measurement of FNA-Tg is still controversial. Some studies have indicated that serum $\mathrm{Tg}-\mathrm{Ab}$ levels could interfere with FNA-Tg measurement and may lower FNA-Tg values. ${ }^{18,27-29}$ Others have reported that circulating Tg-Ab had no significant effect on FNA-Tg measurement. ${ }^{14,17,26,30}$ In recent years, concerns have been raised regarding the possible interference of serum Tg or serum TSH with FNA-Tg measurements. . $^{31,32}$ In our study, no statistically significant differences in preoperative serum $\mathrm{Tg}-\mathrm{Ab}(p=0.322)$ and $\mathrm{TSH}(p=0.907)$ were found between the two groups. Although serum Tg levels ( $p=0.005)$ were significantly different between the two groups, no correlation was found between preoperative serum $\mathrm{Tg}$ and intraoperative FNA-Tg according to Spearman's rank correlation (all patients: $p=0.451$; malignant $\mathrm{LN}$ group: $p=0.248$; benign LN group: $p=0.095$ ). This suggested that higher levels of preoperative serum Tg in patients with PTC might be an indicator of LNM.

For patients with PTMC, the malignancy of LNs is an important factor to determine the extent of surgery. Primary tumor size was shown to be statistically significant in this study between the two groups ( $p=0.000)$. The optimal cutoff value of intraoperative FNA-Tg for the assessment of malignant LNs from PTMCs was analyzed. The optimal cutoff value was $98.1 \mathrm{ng} / \mathrm{mL}$, and the sensitivity and specificity were $84.6 \%$ and $96.2 \%$, respectively. This result might have some auxiliary value to determine the extent of surgery, but more LN samples from PTMCs are needed before further application.
In our study, the aspirated $\mathrm{LN}$ of a female patient was shown to be parathyroid tissue, and the $\mathrm{Tg}$ value was $1.05 \mathrm{ng} / \mathrm{mL}$. This phenomenon is not consistent with the theory that only thyroid follicular epithelial cells can produce Tg. Contamination is a possible explanation. Therefore, it is of great importance to avoid contamination from thyroid tissue or blood when performing FNA-Tg of LN. In addition, it is important to ensure that FNA is performed on the dominant metastatic focus (especially the micrometastatic focus) in order to minimize FN results.

There were several limitations in this study. A selection bias may have existed. In our study, we included the LNs that were firmer, bigger or rounder than the remainder of the available LNs from DTC or suspected DTC patients. Second, the sample size of this study was small, and further studies with more patients will be helpful to validate the cutoff values from the ROC curve. Third, in some cases, it was difficult to distinguish between $\mathrm{LN}$, thyroid tissue, parathyroid tissue and adipose tissue. If FNA-Tg is performed when ectopic thyroid tissue or thyroid nodule is mistaken for suspected malignant LN, then the FNA-Tg level will be high. Thus, an FP result may be obtained. There were two cases of other tissues mistaken for LNs in our study. Further studies are needed to support our present conclusion.

\section{Conclusion}

Intraoperative FNA-Tg level of suspicious metastatic cervical LNs can be useful to diagnose metastatic PTC. The optimal cutoff value was $147.5 \mathrm{ng} / \mathrm{mL}$, and the corresponding sensitivity was superior to that of preoperative ultrasonography. Intraoperative LN-FNA-Tg technique may play an important role in determining the proper surgical procedure.

\section{Acknowledgment}

This study was supported by the National Natural Science Foundation of China (grant number 81672641).

\section{Disclosure}

The authors report no conflicts of interest in this work.

\section{References}

1. Haugen BR, Alexander EK, Bible KC, et al. 2015 American Thyroid Association Management Guidelines for adult patients with thyroid nodules and differentiated thyroid cancer: the American Thyroid Association Guidelines task force on thyroid nodules and differentiated thyroid cancer. Thyroid. 2016;26(1):1-133.

2. Grebe SK, Hay ID. Thyroid cancer nodal metastases: biologic significance and therapeutic considerations. Surg Oncol Clin N Am. 1996; $5(1): 43-63$.

3. Wada N, Duh QY, Sugino K, et al. Lymph node metastasis from 259 papillary thyroid microcarcinomas: frequency, pattern of occurrence and recurrence, and optimal strategy for neck dissection. Ann Surg. 2003; 237(3):399-407. 
4. Yeh MW, Bauer AJ, Bernet VA, et al; American Thyroid Association Surgical Affairs Committee Writing Task Force. American Thyroid Association statement on preoperative imaging for thyroid cancer surgery. Thyroid. 2015;25(1):3-14.

5. Park YM, Wang SG, Goh JY, Shin DH, Kim IJ, Lee BJ. Intraoperative frozen section for the evaluation of extrathyroidal extension in papillary thyroid cancer. World J Surg. 2015;39(1):187-193.

6. Khokhar MT, Day KM, Sangal RB, et al. Preoperative high-resolution ultrasound for the assessment of malignant central compartment lymph nodes in papillary thyroid cancer. Thyroid. 2015;25(12):1351-1354.

7. Solorzano CC, Carneiro DM, Ramirez M, Lee TM, Irvin GL 3rd. Surgeon-performed ultrasound in the management of thyroid malignancy. Am Surg. 2004;70(7):576-580.

8. Jeong HS, Baek CH, Son YI, et al. Integrated 18F-FDG PET/CT for the initial evaluation of cervical node level of patients with papillary thyroid carcinoma: comparison with ultrasound and contrast-enhanced CT. Clin Endocrinol. 2006;65(3):402-407.

9. Suh CH, Baek JH, Choi YJ, Lee JH. Performance of CT in the preoperative diagnosis of cervical lymph node metastasis in patients with papillary thyroid cancer: a systematic review and meta-analysis. AJNR Am J Neuroradiol. 2016;38(1):154-161.

10. Lesnik D, Cunnane ME, Zurakowski D, et al. Papillary thyroid carcinoma nodal surgery directed by a preoperative radiographic map utilizing CT scan and ultrasound in all primary and reoperative patients. Head Neck. 2014;36(2):191-202.

11. Orloff LA, Randolph GW. Preoperative imaging for thyroid cancer: beyond ultrasonography. JAMA Otolaryngol Head Neck Surg. 2016; 142(6):515-516.

12. Pacini F, Fugazzola L, Lippi F, et al. Detection of thyroglobulin in fine needle aspirates of nonthyroidal neck masses: a clue to the diagnosis of metastatic differentiated thyroid cancer. J Clin Endocrinol Metab. 1992;74(6):1401-1404.

13. Frasoldati A, Toschi E, Zini M, et al. Role of thyroglobulin measurement in fine-needle aspiration biopsies of cervical lymph nodes in patients with differentiated thyroid cancer. Thyroid. 1999;9(2):105-111.

14. Baskin HJ. Detection of recurrent papillary thyroid carcinoma by thyroglobulin assessment in the needle washout after fine-needle aspiration of suspicious lymph nodes. Thyroid. 2004;14(11):959-963.

15. Cignarelli M, Ambrosi A, Marino A, et al. Diagnostic utility of thyroglobulin detection in fine-needle aspiration of cervical cystic metastatic lymph nodes from papillary thyroid cancer with negative cytology. Thyroid. 2003;13(12):1163-1167.

16. Uruno T, Miyauchi A, Shimizu K, et al. Usefulness of thyroglobulin measurement in fine-needle aspiration biopsy specimens for diagnosing cervical lymph node metastasis in patients with papillary thyroid cancer. World J Surg. 2005;29(4):483-485.

17. Cunha N, Rodrigues F, Curado F, et al. Thyroglobulin detection in fineneedle aspirates of cervical lymph nodes: a technique for the diagnosis of metastatic differentiated thyroid cancer. Eur J Endocrinol. 2007; 157(1):101-107.

18. Jeon MJ, Park JW, Han JM, et al. Serum antithyroglobulin antibodies interfere with thyroglobulin detection in fine-needle aspirates of metastatic neck nodes in papillary thyroid carcinoma. J Clin Endocrinol Metab. 2013;98(1):153-160.
19. Grani G, Fumarola A. Thyroglobulin in lymph node fine-needle aspiration washout: a systematic review and meta-analysis of diagnostic accuracy. J Clin Endocrinol Metab. 2014;99(6):1970-1982.

20. Makay O, Icoz G, Gurcu B, et al. The ongoing debate in thyroid surgery: should frozen section analysis be omitted? Endocr J. 2007; 54(3):385-390.

21. Peng Y, Wang HH. A meta-analysis of comparing fine-needle aspiration and frozen section for evaluating thyroid nodules. Diagn Cytopathol. 2008;36(12):916-920

22. Prades JM, Querat C, Dumollard JM, et al. Thyroid nodule surgery: predictive diagnostic value of fine-needle aspiration cytology and frozen section. Eur Ann Otorhinolaryngol Head Neck Dis. 2013;130(4): 195-199.

23. Ferris RL, Baloch Z, Bernet V, et al; American Thyroid Association Surgical Affairs Committee. American Thyroid Association statement on surgical application of molecular profiling for thyroid nodules: current impact on perioperative decision making. Thyroid. 2015; 25(7):760-768.

24. Marotta V, Sciammarella C, Capasso M, et al. Germline polymorphisms of the VEGF pathway predict recurrence in nonadvanced differentiated thyroid cancer. J Clin Endocrinol Metab. 2017;102(2):661-671.

25. Conzo G, Avenia N, Ansaldo GL, et al. Surgical treatment of thyroid follicular neoplasms: results of a retrospective analysis of a large clinical series. Endocrine. 2017;55(2):530-538.

26. Boi F, Baghino G, Atzeni F, Lai ML, Faa G, Mariotti S. The diagnostic value for differentiated thyroid carcinoma metastases of thyroglobulin ( $\mathrm{Tg}$ ) measurement in washout fluid from fine-needle aspiration biopsy of neck lymph nodes is maintained in the presence of circulating anti-Tg antibodies. J Clin Endocrinol Metab. 2006;91(4):1364-1369.

27. Jo K, Kim MH, Lim Y, et al. Lowered cutoff of lymph node fine-needle aspiration thyroglobulin in thyroid cancer patients with serum antithyroglobulin antibody. Eur J Endocrinol. 2015;173(4):489-497.

28. Shin HJ, Lee HS, Kim EK, Moon HJ, Lee JH, Kwak JY. A study on serum antithyroglobulin antibodies interference in thyroglobulin measurement in fine-needle aspiration for diagnosing lymph node metastasis in postoperative patients. PLoS One. 2015;10(6):e0131096.

29. Yap NS, Maher R, Learoyd DL. Any detectable thyroglobulin in lymph node biopsy washouts suggests local recurrence in differentiated thyroid cancer. Endocr Connect. 2014;3(4):150-155.

30. Bournaud C, Charrie A, Nozieres C, et al. Thyroglobulin measurement in fine-needle aspirates of lymph nodes in patients with differentiated thyroid cancer: a simple definition of the threshold value, with emphasis on potential pitfalls of the method. Clin Chem Lab Med. 2010; 48(8):1171-1177.

31. Jeon MJ, Kim WG, Jang EK, et al. Thyroglobulin level in fineneedle aspirates for preoperative diagnosis of cervical lymph node metastasis in patients with papillary thyroid carcinoma: two different cutoff values according to serum thyroglobulin level. Thyroid. 2015; 25(4):410-416.

32. Moon JH, Kim YI, Lim JA, et al. Thyroglobulin in washout fluid from lymph node fine-needle aspiration biopsy in papillary thyroid cancer: large-scale validation of the cutoff value to determine malignancy and evaluation of discrepant results. J Clin Endocrinol Metab. 2013 98(3):1061-1068
OncoTargets and Therapy

\section{Publish your work in this journal}

OncoTargets and Therapy is an international, peer-reviewed, open access journal focusing on the pathological basis of all cancers, potential targets for therapy and treatment protocols employed to improve the management of cancer patients. The journal also focuses on the impact of management programs and new therapeutic agents and protocols on

\section{Dovepress}

patient perspectives such as quality of life, adherence and satisfaction. The manuscript management system is completely online and includes a very quick and fair peer-review system, which is all easy to use. Visit http://www.dovepress.com/testimonials.php to read real quotes from published authors. 